\title{
Introduction to the Abstracts of the Invited Talks Presented at ML92 Conference in Aberdeen, 1-3 July 1992
}

D. SLEEM $A N$

SLEEMANGCSD.ABDN.AC. I'K

Department of Computing Science, University of Aberdeen, King's College, Aberdeen AB9 $2 U E$

Embedded in the folklore of our field is the idea that it is essential for every (successful) annual ML conference/workshop to have (three) invited talks: one from a "mainline" ML person and another speaker from a different discipline whose work is seen as being highly relevant to ML. Fortunately the specification of the third speaker is left "fluid."

In my role as Program Chairman for ML92, I discussed the situation with the Organizing Committee and the community. We decided to invite Jude Shavlik to talk on his work that combines symbolic and neural learning; David Klahr, a well-known developmental psychologist, to talk on his empirical work on how children and adults solve tasks which involve discovery; and Ivan Bratko to speak on his work at the intersection of qualitative reasoning and machine learning. (Ivan is also a European, a further constraint I sought to satisfy on the first occasion that the International Machine Learning Conference was held in Europe.)

At the conference, all three talks were well received as clear and informative overviews of their sub-areas. Because none of the invited speakers had papers in the Conference Proceedings (Sleeman \& Edwards, 1992), I approached the Executive Editor of this journal, Tom Dietterich, about publishing extended abstracts for the three talks. It was agreed that each of the abstracts should be sufficient to form a self-contained overview/update on their sub-fields, and so should include a substantial bibliography.

I thank the three Invited Speakers for agreeing readily to undertake an additional postconference task!

\section{References}

Sleeman, D. \& Edwards, P. (1992). Machine Learning: Proceedings of the Ninth International Conference (ML92). San Francisco, CA: Morgan Kaufmann. 